\title{
A Sort-Seq Approach to the Development of Single Fluorescent Protein Biosensors
}

John N. Koberstein ${ }^{1, *}$, Melissa L. Stewart ${ }^{1}$, Taylor L. Mighell ${ }^{2,+}$, Chadwick B. Smith ${ }^{1}$, and Michael S. Cohen ${ }^{3}$

${ }^{1}$ Vollum Institute, Oregon Health \& Science University, Portland, OR 97239, USA.

${ }^{2}$ Department of Molecular and Medical Genetics, Oregon Health \& Science University, Portland, OR 97239, USA

${ }^{3}$ Department of Physiology and Pharmacology, Oregon Health \& Science University, Portland, OR, USA.

+Centre for Genomic Regulation (CRG), 08003 Barcelona, Spain.

*Correspondence: koberste@ohsu.edu (J.N.K.) 


\section{Supplementary Figures}

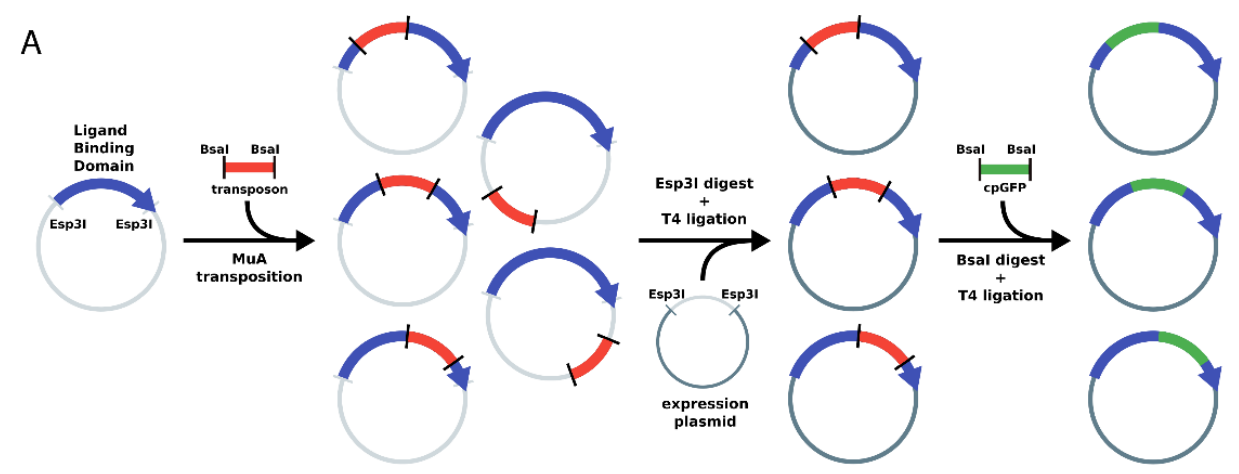

B

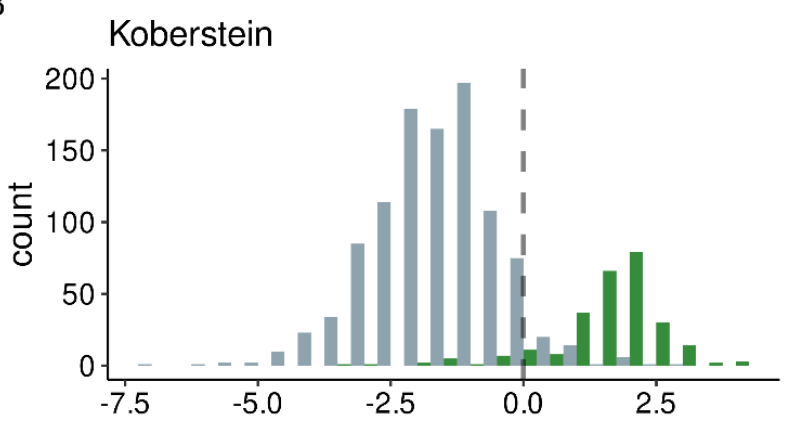

C

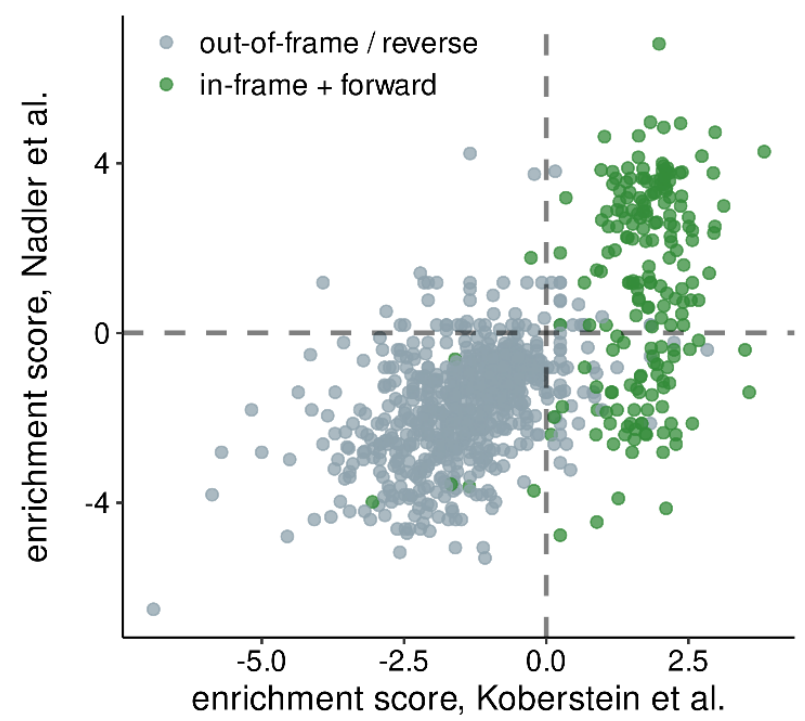

D

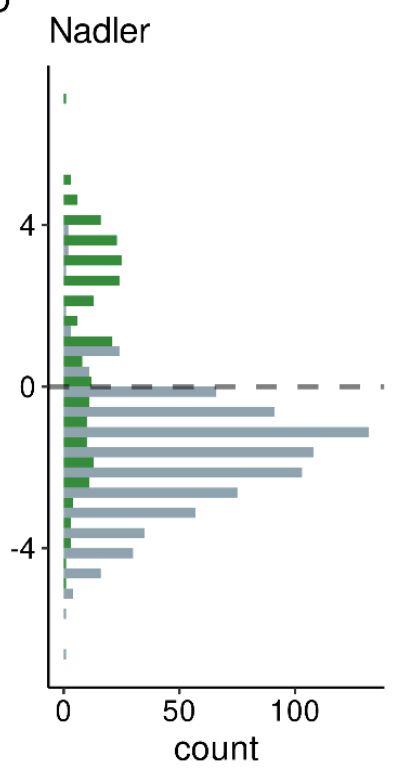

Figure S1. Enrichment of productive cpGFP insertions into MBP. (A) Overview of the transposon mediated domain-insertion cloning method. An engineered transposon sequence is inserted throughout the sequence of a target ligand-binding domain. Subsequent Golden Gate cloning steps move the transposed ORF into an expression plasmid and finally replace the transposon sequence with the coding sequence of cpGFP. Adapted with permission from Nadler, D. C.; Morgan, S.-A.; Flamholz, A.; Kortright, K. E.; Savage, D. F. Rapid Construction of Metabolite Biosensors Using Domain-Insertion Profiling. Nature Communications 2016, 7, 12266. Copyright 2016 Springer Nature. (B) Distribution of enrichment values from this study for MBP domain-insertion variants following a single enrichment sort for GFP+ cells. (C) Comparison of enrichment values for MBP domaininsertion variants across studies reveals consistent enrichment of variants on the basis of fluorescence intensity generated by in-frame and forward insertion of GFP (Spearman's $\rho=0.6$ ). (D) Distribution of enrichment values from Nadler et al. for MBP domain-insertion variants following a single enrichment sort for GFP+ cells. 
A

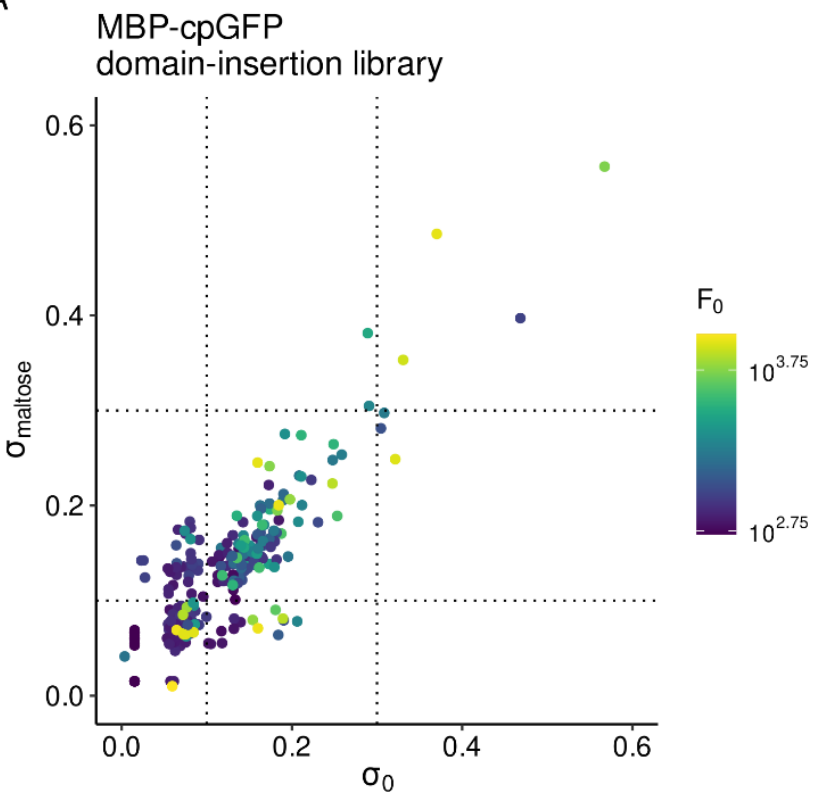

B

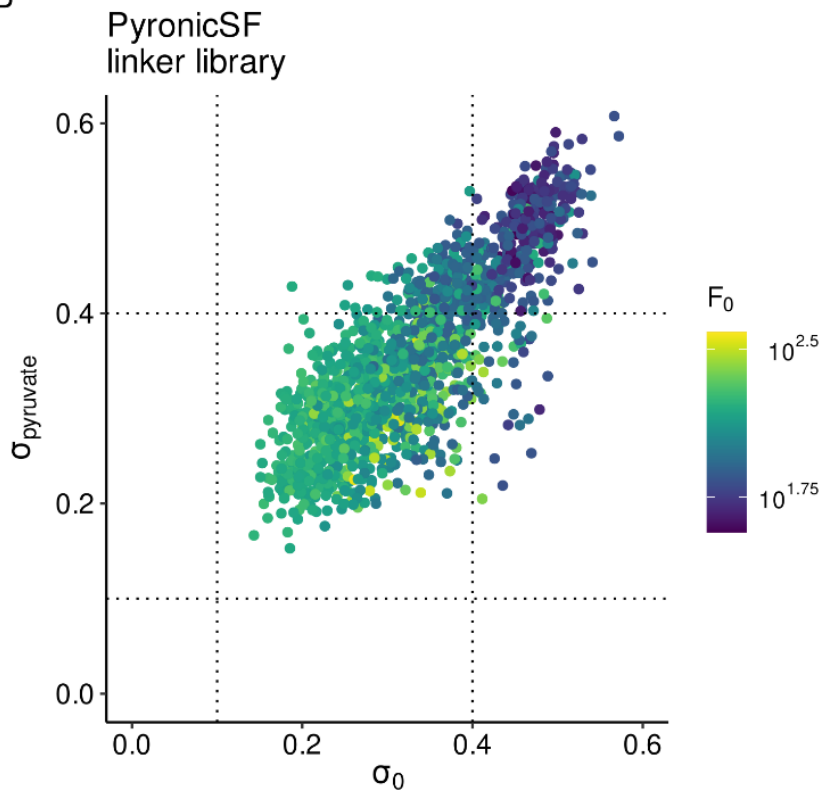

Figure S2. Sort-seq estimates of variance are correlated for individual variants across conditions. (A) Sort-seq derived estimates of log-normal variance $(\sigma)$ compared between conditions for all productive MBP-cpGFP domain-insertion variants. (B) Sort-seq derived estimates of log-normal variance $(\sigma)$ compared between conditions for all PyronicSF linker variants. Dashed lines indicate the lower and upper bounds used to filter out variants with lower and higher variance than expected, respectively. 


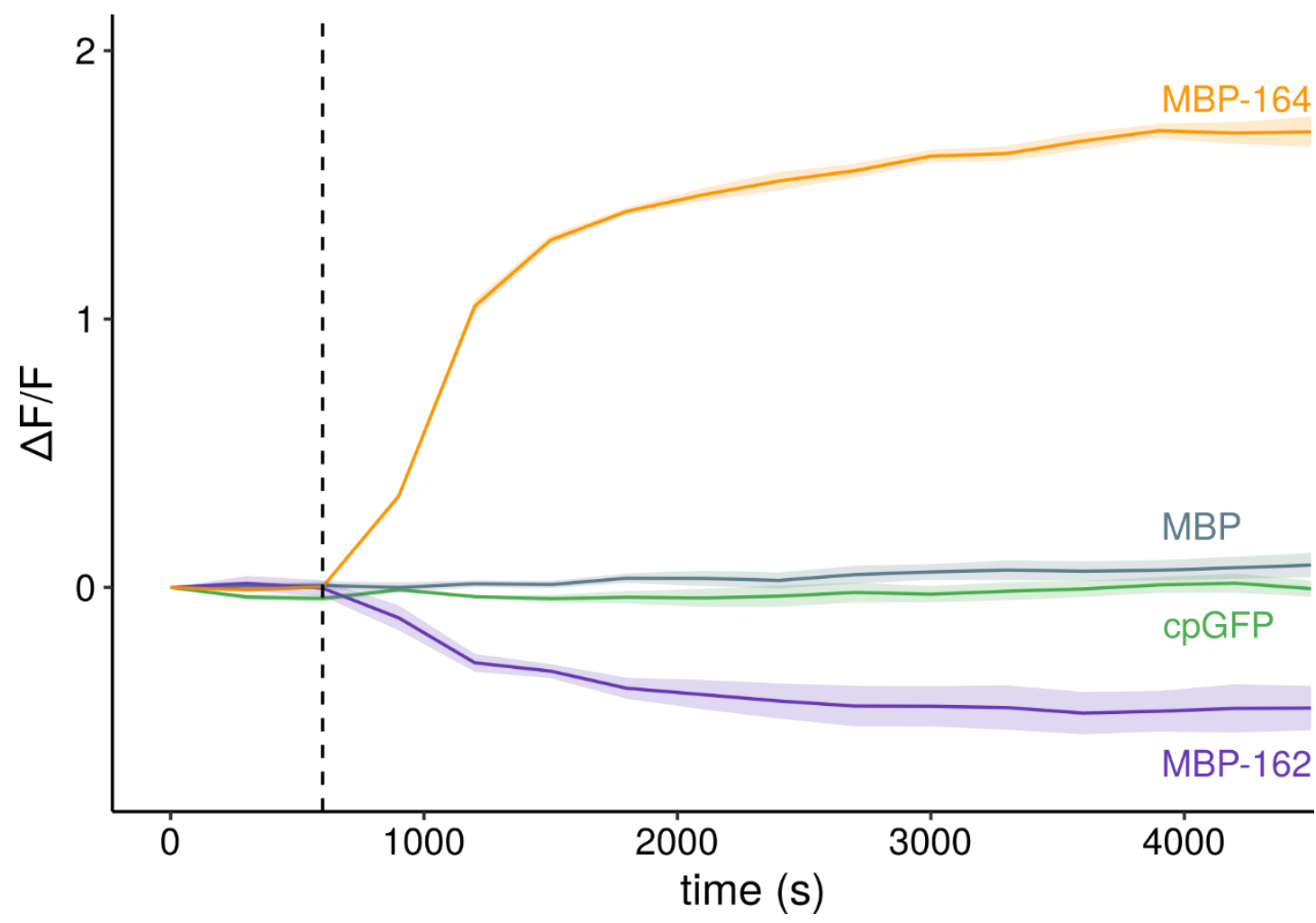

Figure S3. MBP-162 and MBP-164 function as biosensors with opposite responses to increased maltose concentration. The fluorescence of MBP-162 and MBP-164 were monitored while expressed in E. coli following the addition of $1 \mathrm{mM}$ maltose (dashed line) to the media. Controls expressing either MBP or cpGFP were monitored for non-specific changes with addition of maltose. Fluorescence was measured for wells with either maltose or an equal volume of PBS added and the difference between conditions calculated to account for increases in intensity due to changes in protein expression over time. Data are mean \pm s.d. for three replicates for each condition. 


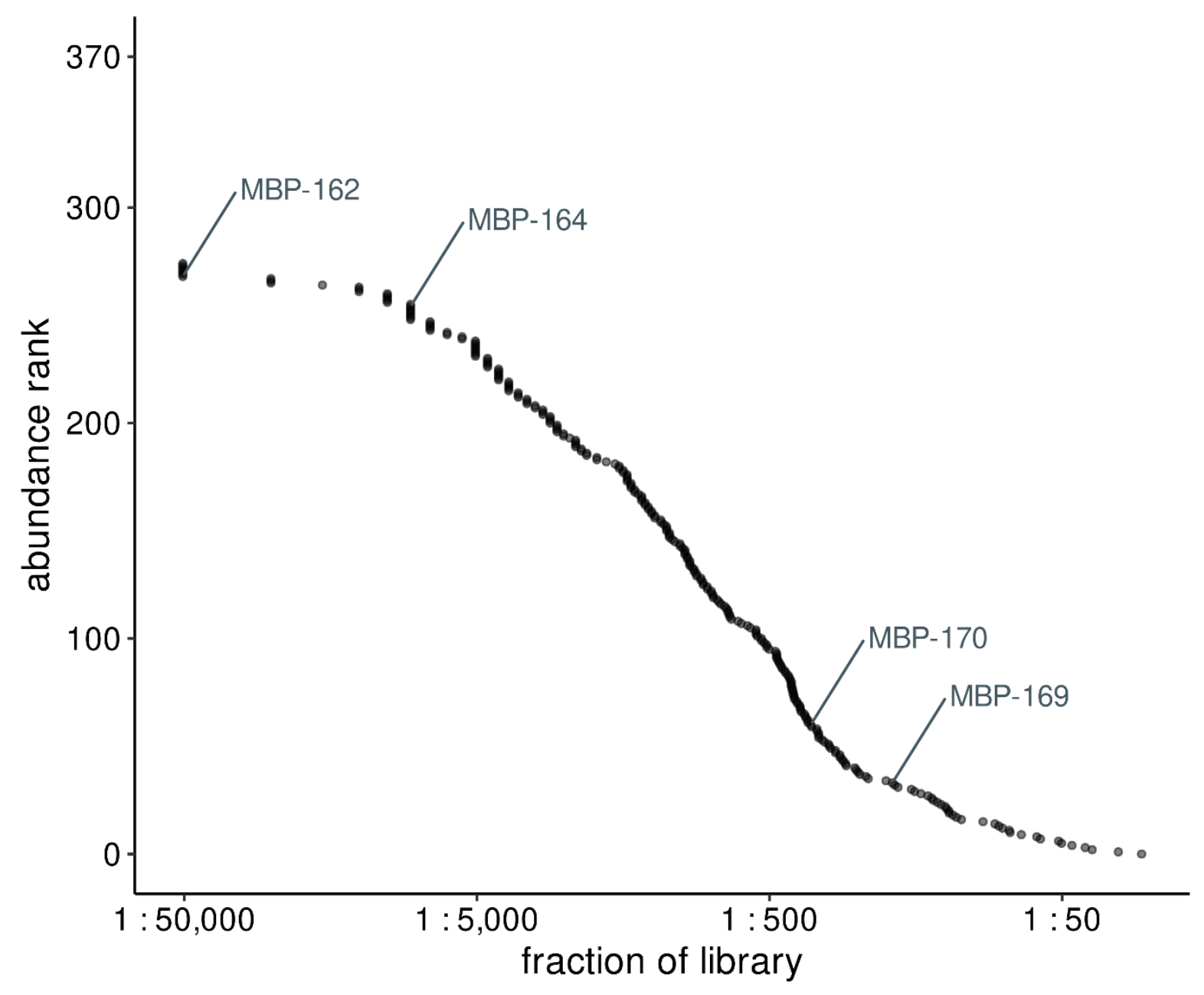

Figure S4. Domain-insertion variant abundance following the fluorescence enrichment sort. The fraction of reads containing a given variant versus the ranked abundance, with 1 being the most abundant variant and 370 being the least. Out of the 370 possible domain-insertion variants, 275 appear at least once in the high-throughput sequencing reads. Abundance is highly variable with some variants appearing only once in approximately 50,000 sequencing reads while others appear as often as once in every 50 reads. 


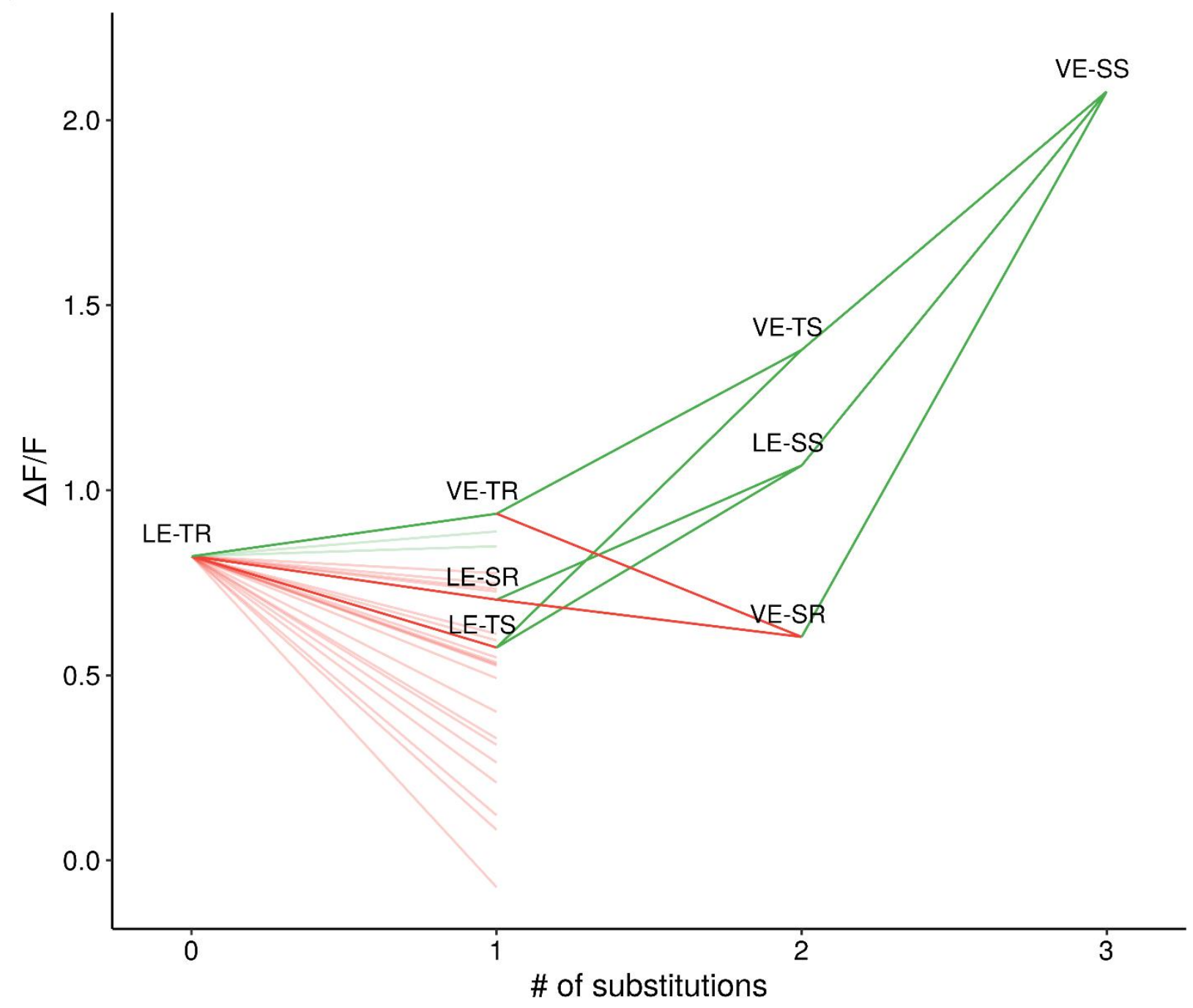

Figure S5. Dynamic range for linker variants versus the number of substitutions to the parent sequence. Five out of the six paths from the parent sequence (LE-TR) to the optimized sequence (VE-SS) travel through intermediates with decreased function (dark lines; green/red indicates increase/decrease in function relative to parent sequence). A majority $(21 / 24)$ of the tested single substitutions to the parent sequence (additional shaded lines) result in a decrease in function. The total increase in dynamic range of VE-SS compared to the parent sequence is much greater $(\Delta \mathrm{F} / \mathrm{F}=2.08)$ than the sum of the effects of the comprising single substitutions $(\Delta \mathrm{F} / \mathrm{F}=0.57)$. 


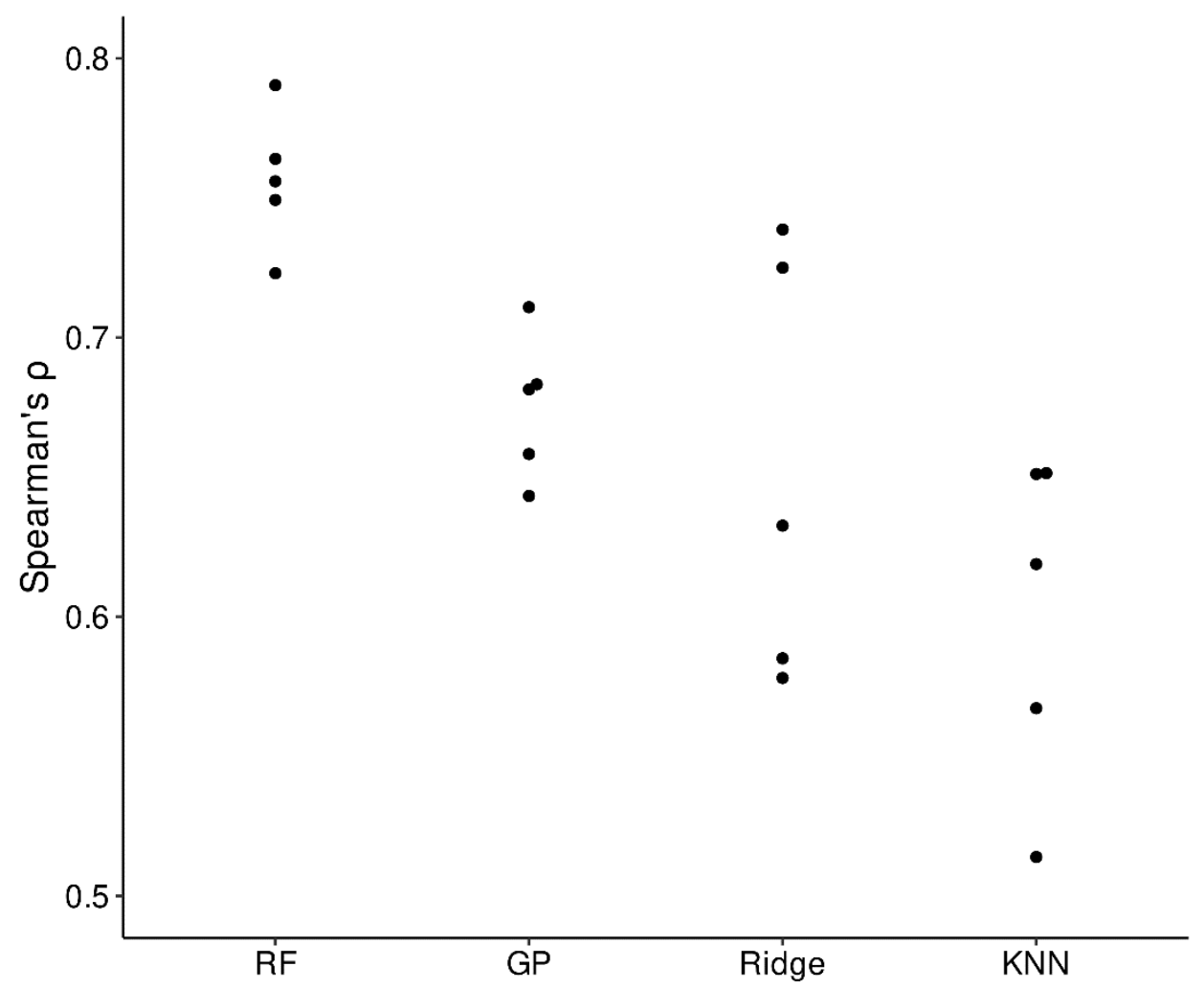

Figure S6. Comparison of different regression models for predicting dynamic range from sequence. Each model was assessed by 5-fold crossvalidation using the Spearman rank correlation coefficient calculated between the model prediction and measured dynamic range for the held-out fold. Random forest exhibits the highest mean correlation $(\rho=0.76)$ across the tested models followed by Gaussian Process (GP, $\rho=0.68)$, ridge regression (Ridge, $\rho=0.65)$ and K-nearest neighbors $(\mathrm{KNN}, \rho=0.60)$. 
A

B

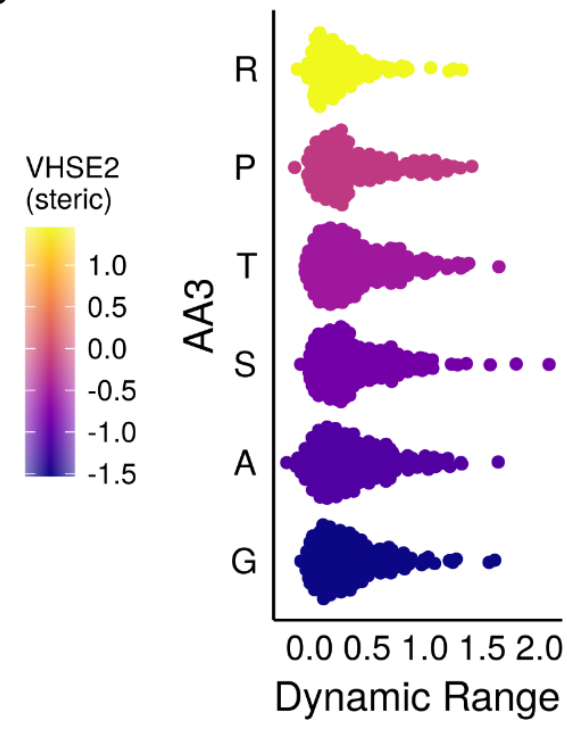

C
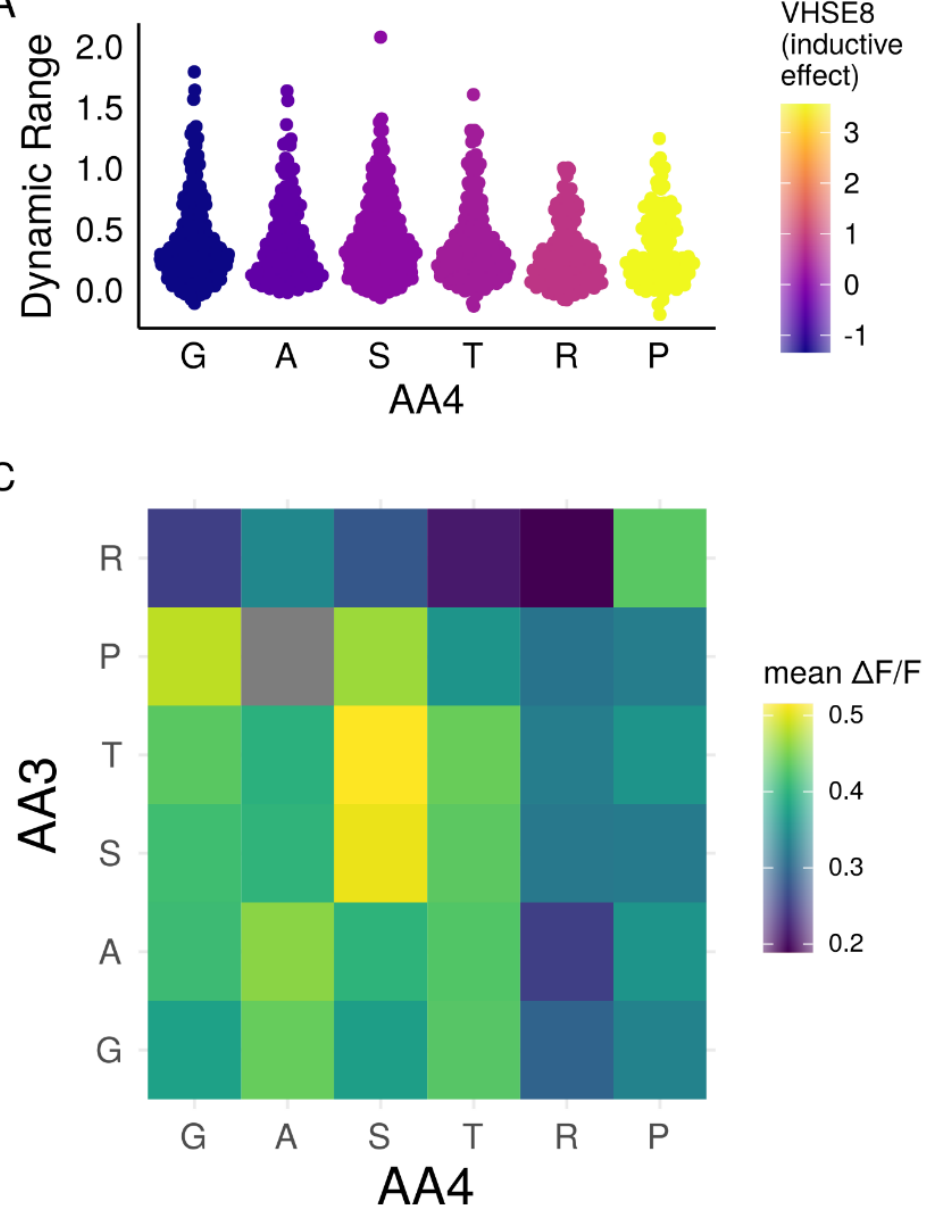

Figure S7. Analysis of the biochemical basis of C-terminal linker function. (A) Distributions of variant dynamic-range estimates sorted by VHSE8 of second C-terminal linker amino acid. (B) Distributions of variant dynamic-range estimates sorted by VHSE2 of the first C-terminal linker amino acid. (C) Mean dynamic-range for C-terminal linker pairs. The combination $\mathrm{P}^{3} \mathrm{~A}^{4}$ is excluded (grey) as no variants with the linker combination passed filtering. 
A

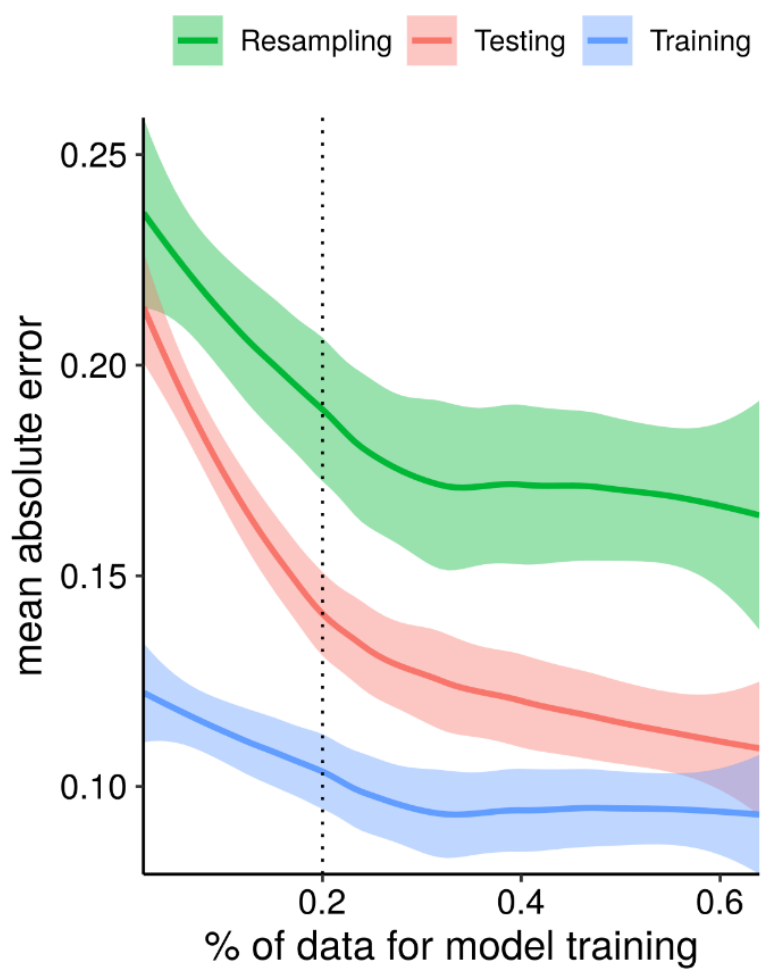

B

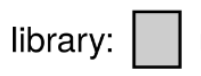
naive predicted high-dynamic-range

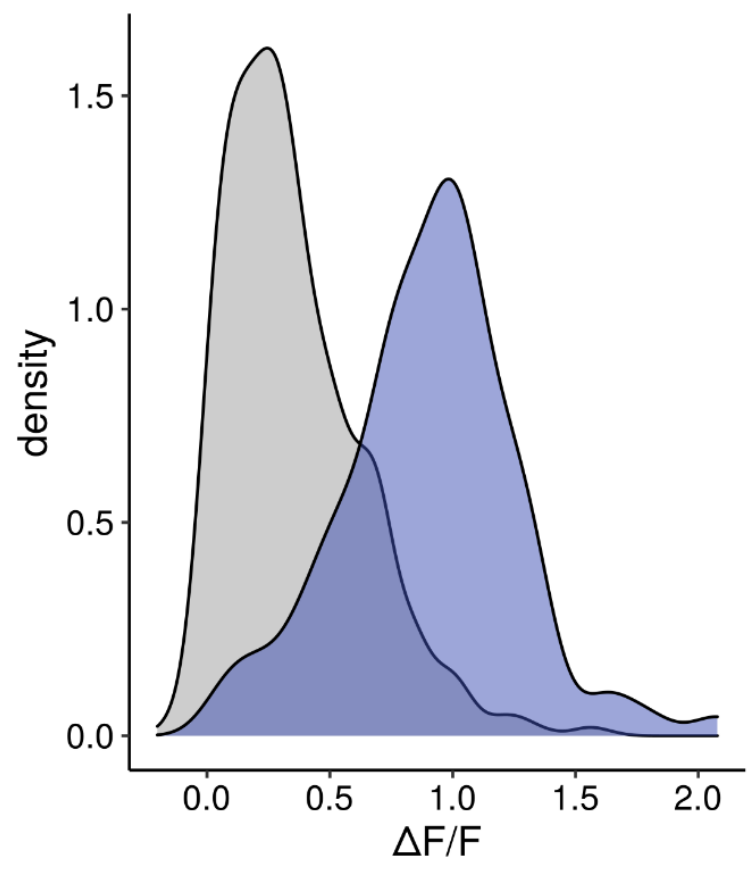

Figure S8. A random forest model trained on a subset of the collected data can be used to design a library with an increase in dynamic range. (A) Mean absolute error as a function of the percent of data used to train the model evaluated on predictions of dynamic range for the test, training, and resampled data. Lines represent a locally weighted smoothing (LOESS) fit and shaded regions represent a 95\% confidence interval. The vertical dashed line represents the $20 \%$ of data used to train the model in panel B. (B) Distribution of dynamic range estimates for variants in the naive library compared to the high-dynamic-range variants predicted by a model trained on $20 \%$ of the data ( 192 variants). 


\section{Supplementary Text. Sequences of the constructs used in this study.}

\section{PyronicSF-LE-TR}

PdhR 1-188

Linkers

cpGFP

PdhR 189-254

Amino Acid:

MGSAYSKIRQPKLSDVIEQQLEFLILEGTLRPGEKLPPERELAKQFDVSRPSLREAIQRLEAKGLLLRRQGGGTFVQSS LWQSFSDPLVELLSDHPESQYDLLETRHALEGIAAYYAALRSTDEDKERIRELHHAIELAQQSGDLDAESNAVLQYQI AVTEAAHNVVLLHLLRCMEPMLAQNVRQNFELLLENVYIKADKQKNGIKANFKIRHNIEDGGVQLAYHYQQNTP IGDGPVLLPDNHYLSVQSKLSKDPNEKRDHMVLLEFVTAAGITLGMDELYKGGTGGSMVSKGEELFTGVVPILVEL DGDVNGHKFSVSGEGEGDATYGKLTLKFICTTGKLPVPWPTLVTTLTYGVQCFSRYPDHMKQHDFFKSAMPEGY IQERTIFFKDDGNYKTRAEVKFEGDTLVNRIELKGIDFKEDGNILGHKLEYNTRYSRREMLPLVSSHRTRIFEAIMAG KPEEAREASHRHLAFIEEILLDRSREESRRERSLRRLEQRKNSG*

\section{DNA:}

ATGGGCAGCGCATATAGCAAAATTCGGCAGCCCAAACTGAGCGATGTGATTGAGCAGCAGCTGGAGTTTCT GATTCTGGAAGGCACCCTGAGGCCTGGAGAGAAACTGCCCCCTGAGCGCGAACTCGCCAAGCAGTTCGAC GTGAGTCGACCATCACTGAGGGAGGCTATCCAGAGGCTGGAAGCAAAGGGACTGCTCCTGAGGAGACAGG GAGGAGGGACTTTCGTGCAGAGCTCCCTGTGGCAGAGCTTCAGCGACCCCCTGGTCGAGCTCCTGTCTGA CCACCCAGAAAGTCAGTACGATCTCCTGGAGACAAGACATGCTCTGGAAGGCATCGCCGCTTACTATGCAG CCCTGCGGTCCACTGACGAGGATAAGGAACGCATCCGAGAGCTGCACCATGCCATTGAACTCGCTCAGCA GTCAGGAGATCTGGATGCAGAGAGCAACGCCGTGCTGCAGTACCAGATTGCAGTCACCGAGGCTGCACAC AATGTGGTCCTCCTGCATCTCCTGAGGTGCATGGAGCCAATGCTGGCCCAGAACGTGAGACAGAATTTTG AGCTCCTGCTCGAAAACGTCTATATCAAGGCCGACAAGCAGAAAAACGGCATTAAGGCTAACTTCAAGATC AGACACAACATCGAGGATGGTGGCGTGCAGCTGGCCTACCATTATCAGCAGAACACACCAATCGGAGATGG ACCAGTGCTGCTCCCAGATAATCACTACCTGAGCGTCCAGTCCAAGCTGTCTAAAGACCCTAACGAGAAGC GGGATCATATGGTGCTGCTCGAATTTGTCACAGCCGCTGGGATCACTCTGGGTATGGACGAGCTCTATAA AGGAGGGACCGGTGGCAGTATGGTGTCAAAGGGCGAGGAACTGTTCACAGGAGTGGTCCCCATTCTGGTG GAGCTCGACGGCGATGTCAATGGACACAAATTTTCCGTGTCTGGCGAGGGCGAAGGAGATGCTACCTACG GGAAGCTGACACTCAAATTCATCTGCACCACAGGCAAGCTGCCAGTGCCCTGGCCTACTCTGGTCACTACC CTCACCTACGGGGTGCAGTGTTTCTCCAGATATCCCGACCACATGAAGCAGCATGATTTCTTTAAATCTGC TATGCCTGAGGGGTACATCCAGGAACGGACAATTTTCTTTAAGGACGATGGTAACTACAAAACACGCGCAG AGGTGAAGTTCGAAGGCGACACTCTGGTCAATCGAATCGAGCTGAAGGGAATTGACTTTAAAGAAGATGGG AACATCCTGGGTCACAAGCTGGAGTACAATACTAGGTATTCTCGGCGCGAAATGCTGCCACTCGTGTCTAG TCACAGGACCAGAATCTTTGAGGCAATTATGGCCGGAAAGCCCGAGGAAGCTAGAGAAGCAAGTCACCGGC ATCTGGCCTTCATCGAGGAAATTCTGCTCGACCGGAGCCGCGAGGAATCCCGAAGGGAGCGCAGCCTGAG GCGACTCGAACAGCGAAAGAACTCAGGCTAA 


\section{PyronicSF-VE-SS}

PdhR 1-188

Linkers

cpGFP

PdhR 189-254

\section{Amino Acid:}

MGSAYSKIRQPKLSDVIEQQLEFLILEGTLRPGEKLPPERELAKQFDVSRPSLREAIQRLEAKGLLLRRQGGGTFVQSS LWQSFSDPLVELLSDHPESQYDLLETRHALEGIAAYYAALRSTDEDKERIRELHHAIELAQQSGDLDAESNAVLQYQI AVTEAAHNVVLLHLLRCMEPMLAQNVRQNFELLVENVYIKADKQKNGIKANFKIRHNIEDGGVQLAYHYQQNTP IGDGPVLLPDNHYLSVQSKLSKDPNEKRDHMVLLEFVTAAGITLGMDELYKGGTGGSMVSKGEELFTGVVPILVEL DGDVNGHKFSVSGEGEGDATYGKLTLKFICTTGKLPVPWPTLVTTLTYGVQCFSRYPDHMKQHDFFKSAMPEGY IQERTIFFKDDGNYKSSAEVKFEGDTLVNRIELKGIDFKEDGNILGHKLEYNTRYSRREMLPLVSSHRTRIFEAIMAG KPEEAREASHRHLAFIEEILLDRSREESRRERSLRRLEQRKNSG*

\section{DNA:}

ATGGGCAGCGCATATAGCAAAATTCGGCAGCCCAAACTGAGCGATGTGATTGAGCAGCAGCTGGAGTTTCT GATTCTGGAAGGCACCCTGAGGCCTGGAGAGAAACTGCCCCCTGAGCGCGAACTCGCCAAGCAGTTCGAC GTGAGTCGACCATCACTGAGGGAGGCTATCCAGAGGCTGGAAGCAAAGGGACTGCTCCTGAGGAGACAGG GAGGAGGGACTTTCGTGCAGAGCTCCCTGTGGCAGAGCTTCAGCGACCCCCTGGTCGAGCTCCTGTCTGA CCACCCAGAAAGTCAGTACGATCTCCTGGAGACAAGACATGCTCTGGAAGGCATCGCCGCTTACTATGCAG CCCTGCGGTCCACTGACGAGGATAAGGAACGCATCCGAGAGCTGCACCATGCCATTGAACTCGCTCAGCA GTCAGGAGATCTGGATGCAGAGAGCAACGCCGTGCTGCAGTACCAGATTGCAGTCACCGAGGCTGCACAC AATGTGGTCCTCCTGCATCTCCTGAGGTGCATGGAGCCAATGCTGGCCCAGAACGTGAGACAGAATTTTG AGCTCCTGGTAGAAAACGTCTATATCAAGGCCGACAAGCAGAAAAACGGCATTAAGGCTAACTTCAAGATC AGACACAACATCGAGGATGGTGGCGTGCAGCTGGCCTACCATTATCAGCAGAACACACCAATCGGAGATGG ACCAGTGCTGCTCCCAGATAATCACTACCTGAGCGTCCAGTCCAAGCTGTCTAAAGACCCTAACGAGAAGC GGGATCATATGGTGCTGCTCGAATTTGTCACAGCCGCTGGGATCACTCTGGGTATGGACGAGCTCTATAA AGGAGGGACCGGTGGCAGTATGGTGTCAAAGGGCGAGGAACTGTTCACAGGAGTGGTCCCCATTCTGGTG GAGCTCGACGGCGATGTCAATGGACACAAATTTTCCGTGTCTGGCGAGGGCGAAGGAGATGCTACCTACG GGAAGCTGACACTCAAATTCATCTGCACCACAGGCAAGCTGCCAGTGCCCTGGCCTACTCTGGTCACTACC CTCACCTACGGGGTGCAGTGTTTCTCCAGATATCCCGACCACATGAAGCAGCATGATTTCTTTAAATCTGC TATGCCTGAGGGGTACATCCAGGAACGGACAATTTTCTTTAAGGACGATGGTAACTACAAAACACGCGCAG AGGTGAAGTTCGAAGGCGACACTCTGGTCAATCGAATCGAGCTGAAGGGAATTGACTTTAAAGAAGATGGG AACATCCTGGGTCACAAGCTGGAGTACAATAGTAGTTATTCTCGGCGCGAAATGCTGCCACTCGTGTCTAG TCACAGGACCAGAATCTTTGAGGCAATTATGGCCGGAAAGCCCGAGGAAGCTAGAGAAGCAAGTCACCGGC ATCTGGCCTTCATCGAGGAAATTCTGCTCGACCGGAGCCGCGAGGAATCCCGAAGGGAGCGCAGCCTGAG GCGACTCGAACAGCGAAAGAACTCAGGCTAA 


\section{PyronicSF-SNAx2/VSTx2}

PdhR 1-188

Linkers

$\mathrm{X}=\{\mathrm{A}, \mathrm{G}, \mathrm{E}, \mathrm{L}, \mathrm{Q}, \mathrm{R}, \mathrm{P}, \mathrm{V}\}$

$\mathrm{Z}=\{\mathrm{A}, \mathrm{G}, \mathrm{P}, \mathrm{R}, \mathrm{S}, \mathrm{T}\}$

cPGFP

PdhR 189-254

\section{Amino Acid:}

MGSAYSKIRQPKLSDVIEQQLEFLILEGTLRPGEKLPPERELAKQFDVSRPSLREAIQRLEAKGLLLRRQGGGTFVQSS LWQSFSDPLVELLSDHPESQYDLLETRHALEGIAAYYAALRSTDEDKERIRELHHAIELAQQSGDLDAESNAVLQYQI AVTEAAHNVVLLHLLRCMEPMLAQNVRQNFELLXXNVYIKADKQKNGIKANFKIRHNIEDGGVQLAYHYQQNTP IGDGPVLLPDNHYLSVQSKLSKDPNEKRDHMVLLEFVTAAGITLGMDELYKGGTGGSMVSKGEELFTGVVPILVEL DGDVNGHKFSVSGEGEGDATYGKLTLKFICTTGKLPVPWPTLVTTLTYGVQCFSRYPDHMKQHDFFKSAMPEGY IQERTIFFKDDGNYKZZAEVKFEGDTLVNRIELKGIDFKEDGNILGHKLEYNTRYSRREMLPLVSSHRTRIFEAIMAG KPEEAREASHRHLAFIEEILLDRSREESRRERSLRRLEQRKNSG*

\section{DNA:}

ATGGGCAGCGCATATAGCAAAATTCGGCAGCCCAAACTGAGCGATGTGATTGAGCAGCAGCTGGAGTTTCT GATTCTGGAAGGCACCCTGAGGCCTGGAGAGAAACTGCCCCCTGAGCGCGAACTCGCCAAGCAGTTCGAC GTGAGTCGACCATCACTGAGGGAGGCTATCCAGAGGCTGGAAGCAAAGGGACTGCTCCTGAGGAGACAGG GAGGAGGGACTTTCGTGCAGAGCTCCCTGTGGCAGAGCTTCAGCGACCCCCTGGTCGAGCTCCTGTCTGA CCACCCAGAAAGTCAGTACGATCTCCTGGAGACAAGACATGCTCTGGAAGGCATCGCCGCTTACTATGCAG CCCTGCGGTCCACTGACGAGGATAAGGAACGCATCCGAGAGCTGCACCATGCCATTGAACTCGCTCAGCA GTCAGGAGATCTGGATGCAGAGAGCAACGCCGTGCTGCAGTACCAGATTGCAGTCACCGAGGCTGCACAC AATGTGGTCCTCCTGCATCTCCTGAGGTGCATGGAGCCAATGCTGGCCCAGAACGTGAGACAGAATTTTG AGCTCCTGSNASNAAACGTCTATATCAAGGCCGACAAGCAGAAAAACGGCATTAAGGCTAACTTCAAGATCA GACACAACATCGAGGATGGTGGCGTGCAGCTGGCCTACCATTATCAGCAGAACACACCAATCGGAGATGGA CCAGTGCTGCTCCCAGATAATCACTACCTGAGCGTCCAGTCCAAGCTGTCTAAAGACCCTAACGAGAAGCG GGATCATATGGTGCTGCTCGAATTTGTCACAGCCGCTGGGATCACTCTGGGTATGGACGAGCTCTATAAA GGAGGGACCGGTGGCAGTATGGTGTCAAAGGGCGAGGAACTGTTCACAGGAGTGGTCCCCATTCTGGTGG AGCTCGACGGCGATGTCAATGGACACAAATTTTCCGTGTCTGGCGAGGGCGAAGGAGATGCTACCTACGG GAAGCTGACACTCAAATTCATCTGCACCACAGGCAAGCTGCCAGTGCCCTGGCCTACTCTGGTCACTACCC TCACCTACGGGGTGCAGTGTTTCTCCAGATATCCCGACCACATGAAGCAGCATGATTTCTTTAAATCTGCT ATGCCTGAGGGGTACATCCAGGAACGGACAATTTTCTTTAAGGACGATGGTAACTACAAAACACGCGCAGA GGTGAAGTTCGAAGGCGACACTCTGGTCAATCGAATCGAGCTGAAGGGAATTGACTTTAAAGAAGATGGGA ACATCCTGGGTCACAAGCTGGAGTACAATVSTVSTTATTCTCGGCGCGAAATGCTGCCACTCGTGTCTAGT CACAGGACCAGAATCTTTGAGGCAATTATGGCCGGAAAGCCCGAGGAAGCTAGAGAAGCAAGTCACCGGCA TCTGGCCTTCATCGAGGAAATTCTGCTCGACCGGAGCCGCGAGGAATCCCGAAGGGAGCGCAGCCTGAGG CGACTCGAACAGCGAAAGAACTCAGGCTAA 


\section{$\underline{\text { MBP-162 }}$}

MBP 1-162

Linkers

cpGFP

MBP $161-370$

Amino Acid:

MSKIEEGKLVIWINGDKGYNGLAEVGKKFEKDTGIKVTVEHPDKLEEKFPQVAATGDGPDIIFWAHDRFGGYAQS GLLAEITPDKAFQDKLYPFTWDAVRYNGKLIAYPIAVEALSLIYNKDLLPNPPKTWEEIPALDKELKAKGKSALMFNL QEPYFTWPLIAASYNVFIMADKQKNGIKANFKIRHNIEDGGVQLAYHYQQNTPIGDGPVLLPDNHYLSVQSKLSKD PNEKRDHMVLLEFVTAAGITLGMDELYKGGTGGSMVSKGEELFTGVVPILVELDGDVNGHKFSVSGEGEGDATY GKLTLKFICTTGKLPVPWPTLVTTLTYGVQCFSRYPDHMKQHDFFKSAMPEGYIQERTIFFKDDGNYKTRAEVKF EGDTLVNRIELKGIDFKEDGNILGHKLEYNFNASIAADGGYAFKYENGKYDIKDVGVDNAGAKAGLTFLVDLIKNK HMNADTDYSIAEAAFNKGETAMTINGPWAWSNIDTSKVNYGVTVLPTFKGQPSKPFVGVLSAGINAASPNKELAK EFLENYLLTDEGLEAVNKDKPLGAVALKSYEEELAKDPRIAATMENAQKGEIMPNIPQMSAFWYAVRTAVINAASG RQTVDEALKDAQTRITKSGHHHHHH*

\section{DNA:}

ATGTCCAAAATCGAAGAAGGTAAACTGGTAATCTGGATTAACGGCGATAAAGGCTATAACGGACTCGCTGA AGTCGGTAAGAAATTCGAGAAAGATACCGGAATTAAAGTCACCGTTGAGCATCCGGATAAACTGGAAGAGA AATTCCCACAGGTTGCGGCAACTGGCGATGGCCCTGACATTATCTTCTGGGCACACGACCGCTTTGGTGG CTACGCTCAATCTGGCCTGTTGGCTGAAATCACCCCGGACAAAGCGTTCCAGGACAAGCTGTATCCGTTTA CCTGGGATGCCGTACGTTACAACGGCAAGCTGATTGCTTACCCGATCGCTGTTGAAGCGTTATCGCTGAT TTATAACAAAGATCTGCTGCCGAACCCGCCAAAAACCTGGGAAGAGATCCCGGCGCTGGATAAAGAACTGA AAGCGAAAGGTAAGAGCGCGCTGATGTTCAACCTGCAAGAACCGTACTTCACCTGGCCGCTGATTGCTGCA TCTTATAACGTCTTTATCATGGCCGACAAGCAGAAGAACGGCATCAAGGCGAACTTCAAGATCCGCCACAA CATCGAGGACGGCGGCGTGCAGCTCGCCTATCACTACCAGCAGAACACCCCCATCGGCGACGGCCCCGTG CTGCTGCCCGACAACCACTACCTGAGCGTGCAGTCCAAACTGAGCAAAGACCCCAACGAGAAGCGCGATCA CATGGTCCTGCTGGAGTTCGTGACCGCCGCCGGGATCACTCTCGGCATGGACGAGCTGTACAAGGGCGGT ACCGGAGGGAGCATGGTGAGCAAGGGCGAGGAGCTGTTCACCGGGGTGGTGCCCATCCTGGTCGAGCTG GACGGCGACGTAAACGGCCACAAGTTCAGCGTGTCCGGCGAGGGCGAGGGCGATGCCACCTACGGCAAGC TGACCCTGAAGTTCATCTGCACCACCGGCAAGCTGCCCGTGCCCTGGCCCACCCTCGTGACCACCCTGAC CTACGGCGTGCAGTGCTTCAGCCGCTACCCCGACCACATGAAGCAGCACGACTTCTTCAAGTCCGCCATG CCCGAAGGCTACATTCAGGAGCGCACCATCTTCTTCAAGGACGACGGCAACTATAAGACACGCGCTGAGGT TAAGTTCGAGGGCGACACTCTGGTTAACCGCATCGAGCTGAAGGGCATCGACTTCAAGGAGGACGGCAAC ATCCTGGGCCATAAGCTTGAATATAACTTCAACGCGTCAATTGCTGCTGACGGGGGTTATGCGTTCAAGTA TGAAAACGGCAAGTACGACATTAAAGACGTGGGCGTGGATAACGCTGGCGCGAAAGCGGGTCTGACCTTC CTGGTTGACCTGATTAAAAACAAACACATGAATGCAGACACCGATTACTCCATCGCAGAAGCTGCCTTTAAT AAAGGCGAAACAGCGATGACCATCAACGGCCCGTGGGCATGGTCCAACATCGACACCAGCAAAGTGAATTA TGGTGTAACGGTACTGCCGACCTTCAAGGGTCAACCATCCAAACCGTTCGTTGGCGTGCTGAGCGCAGGT ATTAACGCCGCCAGTCCGAACAAAGAGCTGGCGAAAGAGTTCCTCGAAAACTATCTGCTGACTGATGAAGG TCTGGAAGCGGTTAATAAAGACAAACCGCTGGGTGCCGTAGCGCTGAAGTCTTACGAGGAAGAGTTGGCG AAAGATCCACGTATTGCCGCCACCATGGAAAACGCCCAGAAAGGTGAAATCATGCCGAACATCCCGCAGAT GTCCGCTTTCTGGTATGCCGTGCGTACTGCGGTGATCAACGCCGCCAGCGGTCGTCAGACTGTCGATGAA GCCCTGAAAGACGCGCAGACTCGTATCACCAAGAGCGGTCACCATCACCATCACCATTAA 


\section{$\underline{\text { MBP-164 }}$}

MBP 1-164

Linkers

cpGFP

MBP 163-370

\section{Amino Acid:}

MSKIEEGKLVIWINGDKGYNGLAEVGKKFEKDTGIKVTVEHPDKLEEKFPQVAATGDGPDIIFWAHDRFGGYAQS GLLAEITPDKAFQDKLYPFTWDAVRYNGKLIAYPIAVEALSLIYNKDLLPNPPKTWEEIPALDKELKAKGKSALMFNL QEPYFTWPLIAADASYNVFIMADKQKNGIKANFKIRHNIEDGGVQLAYHYQQNTPIGDGPVLLPDNHYLSVQSKLS KDPNEKRDHMVLLEFVTAAGITLGMDELYKGGTGGSMVSKGEELFTGVVPILVELDGDVNGHKFSVSGEGEGDA TYGKLTLKFICTTGKLPVPWPTLVTTLTYGVQCFSRYPDHMKQHDFFKSAMPEGYIQERTIFFKDDGNYKTRAEV KFEGDTLVNRIELKGIDFKEDGNILGHKLEYNFNASADGGYAFKYENGKYDIKDVGVDNAGAKAGLTFLVDLIKNK HMNADTDYSIAEAAFNKGETAMTINGPWAWSNIDTSKVNYGVTVLPTFKGQPSKPFVGVLSAGINAASPNKELAK EFLENYLLTDEGLEAVNKDKPLGAVALKSYEEELAKDPRIAATMENAQKGEIMPNIPQMSAFWYAVRTAVINAASG RQTVDEALKDAQTRITKSGHHHHHH*

\section{DNA:}

ATGTCCAAAATCGAAGAAGGTAAACTGGTAATCTGGATTAACGGCGATAAAGGCTATAACGGACTCGCTGA AGTCGGTAAGAAATTCGAGAAAGATACCGGAATTAAAGTCACCGTTGAGCATCCGGATAAACTGGAAGAGA AATTCCCACAGGTTGCGGCAACTGGCGATGGCCCTGACATTATCTTCTGGGCACACGACCGCTTTGGTGG CTACGCTCAATCTGGCCTGTTGGCTGAAATCACCCCGGACAAAGCGTTCCAGGACAAGCTGTATCCGTTTA CCTGGGATGCCGTACGTTACAACGGCAAGCTGATTGCTTACCCGATCGCTGTTGAAGCGTTATCGCTGAT TTATAACAAAGATCTGCTGCCGAACCCGCCAAAAACCTGGGAAGAGATCCCGGCGCTGGATAAAGAACTGA AAGCGAAAGGTAAGAGCGCGCTGATGTTCAACCTGCAAGAACCGTACTTCACCTGGCCGCTGATTGCTGC TGATGCATCTTATAACGTCTTTATCATGGCCGACAAGCAGAAGAACGGCATCAAGGCGAACTTCAAGATCC GCCACAACATCGAGGACGGCGGCGTGCAGCTCGCCTATCACTACCAGCAGAACACCCCCATCGGCGACGG CCCCGTGCTGCTGCCCGACAACCACTACCTGAGCGTGCAGTCCAAACTGAGCAAAGACCCCAACGAGAAG CGCGATCACATGGTCCTGCTGGAGTTCGTGACCGCCGCCGGGATCACTCTCGGCATGGACGAGCTGTACA AGGGCGGTACCGGAGGGAGCATGGTGAGCAAGGGCGAGGAGCTGTTCACCGGGGTGGTGCCCATCCTGG TCGAGCTGGACGGCGACGTAAACGGCCACAAGTTCAGCGTGTCCGGCGAGGGCGAGGGCGATGCCACCTA CGGCAAGCTGACCCTGAAGTTCATCTGCACCACCGGCAAGCTGCCCGTGCCCTGGCCCACCCTCGTGACC ACCCTGACCTACGGCGTGCAGTGCTTCAGCCGCTACCCCGACCACATGAAGCAGCACGACTTCTTCAAGT CCGCCATGCCCGAAGGCTACATTCAGGAGCGCACCATCTTCTTCAAGGACGACGGCAACTATAAGACACGC GCTGAGGTTAAGTTCGAGGGCGACACTCTGGTTAACCGCATCGAGCTGAAGGGCATCGACTTCAAGGAGG ACGGCAACATCCTGGGCCATAAGCTTGAATATAACTTCAACGCGTCAGCTGACGGGGGTTATGCGTTCAAG TATGAAAACGGCAAGTACGACATTAAAGACGTGGGCGTGGATAACGCTGGCGCGAAAGCGGGTCTGACCT TCCTGGTTGACCTGATTAAAAACAAACACATGAATGCAGACACCGATTACTCCATCGCAGAAGCTGCCTTT AATAAAGGCGAAACAGCGATGACCATCAACGGCCCGTGGGCATGGTCCAACATCGACACCAGCAAAGTGAA TTATGGTGTAACGGTACTGCCGACCTTCAAGGGTCAACCATCCAAACCGTTCGTTGGCGTGCTGAGCGCA GGTATTAACGCCGCCAGTCCGAACAAAGAGCTGGCGAAAGAGTTCCTCGAAAACTATCTGCTGACTGATGA AGGTCTGGAAGCGGTTAATAAAGACAAACCGCTGGGTGCCGTAGCGCTGAAGTCTTACGAGGAAGAGTTG GCGAAAGATCCACGTATTGCCGCCACCATGGAAAACGCCCAGAAAGGTGAAATCATGCCGAACATCCCGCA GATGTCCGCTTTCTGGTATGCCGTGCGTACTGCGGTGATCAACGCCGCCAGCGGTCGTCAGACTGTCGAT GAAGCCCTGAAAGACGCGCAGACTCGTATCACCAAGAGCGGTCACCATCACCATCACCATTAA 


\section{EMMA-attB-Dest}

\section{Source:}

HC_Kan_attB-p1

YCe2982 HC_Kan_conn_B-E

YCe3756 HC_Kan_conn_E-F

YCe1895 HC_Kan_Kozak-ATG_p6

YCe1695 HC_Kan_RFP-p7 (BsmbI sites)
YCe1921 HC_Kan_conn_H-K

YCe1889 HC_Kan_SV40-polyA_p11

YCe2731 HC_Kan_conn_L-W

YCe2721 HC_Kan_conn_W-Z

YCe3736_HC_Amp_ccdB_receiver vector

\section{Sequence:}

TAGGAAGGCTTGTCGACGACGGCGGTCTCCGTCGTCAGGATCATCGATGGACTAACTACGGTGAAAGAGACTTAGAAG GAGATTGAGAGATCCGTTAGCCTTTAGTGAACCGTCAGAATTAATTCAGATCGATCTACCAGAACCGTCAGATCCGCTA GAGATTACGCCAACCGCCACCATGGGCAGCAGAGACGGAATTCGCGGCCGCTTCTAGAGCAATACGCAAACCGCCTCT CCCCGCGCGTTGGCCGATTCATTAATGCAGCTGGCACGACAGGTTTCCCGACTGGAAAGCGGGCAGTGAGCGCAACGC AATTAATGTGAGTTAGCTCACTCATTAGGCACCCCAGGCTTTACACTTTATGCTTCCGGCTCGTATGTTGTGTGGAATT GTGAGCGGATAACAATTTCACACATACTAGAGAAAGAGGAGAAATACTAGATGGCTTCCTCCGAAGACGTTATCAAAGA GTTCATGCGTTTCAAAGTTCGTATGGAAGGTTCCGTTAACGGTCACGAGTTCGAAATCGAAGGTGAAGGTGAAGGTCG TCCGTACGAAGGTACCCAGACCGCTAAACTGAAAGTTACCAAAGGTGGTCCGCTGCCGTTCGCTTGGGACATCCTGTC CCCGCAGTTCCAGTACGGTTCCAAAGCTTACGTTAAACACCCGGCTGACATCCCGGACTACCTGAAACTGTCCTTCCC GGAAGGTTTCAAATGGGAACGTGTTATGAACTTCGAAGACGGTGGTGTTGTTACCGTTACCCAGGACTCCTCCCTGCA AGACGGTGAGTTCATCTACAAAGTTAAACTGCGTGGTACCAACTTCCCGTCCGACGGTCCGGTTATGCAGAAAAAAACC ATGGGTTGGGAAGCTTCCACCGAACGTATGTACCCGGAAGACGGTGCTCTGAAAGGTGAAATCAAAATGCGTCTGAAAC TGAAAGACGGTGGTCACTACGACGCTGAAGTTAAAACCACCTACATGGCTAAAAAACCGGTTCAGCTGCCGGGTGCTTA CAAAACCGACATCAAACTGGACATCACCTCCCACAACGAAGACTACACCATCGTTGAACAGTACGAACGTGCTGAAGGT CGTCACTCCACCGGTGCTTAATAACGCTGATAGTGCTAGTGTAGATCGCTACTAGAGCCAGGCATCAAATAAAACGAAA GGCTCAGTCGAAAGACTGGGCCTTTCGTTTTATCTGTTGTTTGTCGGTGAACGCTCTCTACTAGAGTCACACTGGCTC ACCTTCGGGTGGGCCTTTCTGCGTTTATATACTAGTAGCGGCCGCTGCAGCGTCTCTAGGCTAATAACAGCTTCCGGA CTCTAGAACATCCCTACAGGTGATATCCTCGGGTAACTTGTTTATTGCAGCTTATAATGGTTACAAATAAAGCAATAGCA TCACAAATTTCACAAATAAAGCATTTTTTTCACTGCATTCTAGTTGTGGTTTGTCCAAACTCATCAATGTATCTTATCAT GTCTGTCGTCTATGATGAGGATGTTGGTGGAGAGCATGTGGAGGAAGTGGATAGGGAAGGTTGTAGAGTAGATCCGGT TGAAGTGATGAGGATAGGAGGAGGCGAACACATATTGATCCTCGTCACAACCACACCAGCACACCTCAAATCCCACACC ACTCCCACAATTACCATTCACTCAACAAACTCACACATCCCACGATAACGAATTCAAGCTTGATATCATTCAGGACGAGC CTCAGACTCCAGCGTAACTGGACTGCAATCAACTCACTGGCTCACCTTCACGGGTGGGCCTTTCTTCGGTAGAAAATCA AAGGATCTTCTTGAGATCCTTTTTTTCTGCGCGTAATCTGCTGCTTGCAAACAAAAAAACCACCGCTACCAGCGGTGGT TTGTTTGCCGGATCAAGAGCTACCAACTCTTTTTCCGAGGTAACTGGCTTCAGCAGAGCGCAGATACCAAATACTGTTC TTCTAGTGTAGCCGTAGTTAGGCCACCACTTCAAGAACTCTGTAGCACCGCCTACATACCTCGCTCTGCTAATCCTGTT ACCAGTGGCTGCTGCCAGTGGCGATAAGTCGTGTCTTACCGGGTTGGACTCAAGACGATAGTTACCGGATAAGGCGCA GCGGTCGGGCTGAACGGGGGGTTCGTGCACACAGCCCAGCTTGGAGCGAACGACCTACACCGAACTGAGATACCTACA GCGTGAGCTATGAGAAAGCGCCACGCTTCCCGAAGGGAGAAAGGCGGACAGGTATCCGGTAAGCGGCAGGGTCGGAAC AGGAGAGCGCACGAGGGAGCTTCCAGGGGGAAACGCCTGGTATCTTTATAGTCCTGTCGGGTTTCGCCACCTCTGACT TGAGCATCGATTTTTGTGATGCTCGTCAGGGGGGCGGAGCCTATGGAAAAACGCCAGCAACGCAGAAAGGCCCACCCG AAGGTGAGCCAGGTGATTACATTTGGGCCCTCATTACCAATGCTTAATCAGTGAGGCACCTATCTCAGCGATCTGTCTA TTTCGTTCATCCATAGTTGCCTGACTCCCCGTCGTGTAGATAACTACGATGCGGGAGGGCTTACCATCTGGCCCCAGT GCTGCAATGATACCGCGAGAACCACGCTCACCGGCTCCAGATTTATCAGCAATAAACCAGCCAGCCGGGAGGGCCGAG CGCAGAAGTGATCCTGCAACTTTATCCGCCTCCATCCAGTCTATTAATTGTTGCCGGGAAGCTAGAGTAAGTAGTTCGC CAGTTAATAGTTTGCGCAACGTTGTTGCCATTGCTACAGGCATCGTGGTGTCACGCTCGTCGTTTGGTATGGCTTCAT TCAGCTCCGGTTCCCAACGATCAAGGCGAGTTACATGATCCCCCATGTTGTGCAAAAAAGCGGTTAGCTCCTTCGGTC CTCCGATCGTTGCCAGAAGTAAGTTGGCCGCAGTGTTATCACTCATGGTTATGGCAGCACTGCATAATTCTCTTACTGT CATGCCATCCGTGAGATGCTTTTCTGTGACTGGTGAGTACTCAACCAAGTCATTCTGAGAATAGTGTATGCGGCGACC 
GAGTTGCTCTTGCCCGGCGTCAATACGGGATAATACCGCGCCACATAGCAGAACTTTAAAAGTGCTCATCATTGGAAAA CGTTCTTCGGGGCGTAAACTCTCAAGGATCTTACCGCTGTTGAGATCCAGTTCGATGTAACCCACTCGTGCACCCAAC TGATCTTCAGCATCTTTTACTTTCACCAGCGTTTCTGGGTGAGCAAAAACAGGAAGGCAAAATGCCGCAAAAAAGGGAA TAAGGGCGACACGGAAATGTTGAATACTCATTTTAGCTTCCTTAGCTCCTGAAAATCTCGATAACTCAAAAAATACGCCC GGTAGTGATCTTATTTCATTATGGTGAAAGTTGGAACCTCTTACGTGCCGATCAAGTCAAAAGCCTCCGGTCGGAGGCT TTTGACTTTCTGCTATGGAGGTCAGGTATGATTTAAATGGTCAGTATTGAGCGATATCTAGAGAATTCGTCA 


\section{Supplementary Table 1}

\begin{tabular}{|c|c|c|}
\hline Type & Name & Sequence \\
\hline \multirow{20}{*}{ 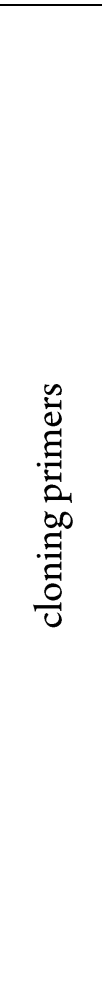 } & MBP-BsaI-GG-F & cacaccaggtctcaGTCCAAAATCGAAGAAGGTAAACTGGTAATCTGG \\
\hline & MBP-BsaI-GG-R & cacaccaggtctcaCGCTCTTGGTGATACGAGTCTGCGC \\
\hline & cpGFP-BsaI-GG-F & caatgcggtctcgcatcTATAACGTCTTTATCATGGC \\
\hline & cpGFP-BsaI-GG-R & caatgcggtctcgacgcGTTGAAGTTATATTCAAGCT \\
\hline & MBP-162-GA-F1 & AATATAACTTCAACGCGTCAATTGCTGCTGACGGGGGTTA \\
\hline & MBP-162-GA-R1 & TAAAGACGTTATAAGATGCAGCAATCAGCGGCCAGGTGAA \\
\hline & MBP-162-GA-F2 & TTCACCTGGCCGCTGATTGCTGCATCTTATAACGTCTTTA \\
\hline & MBP-162-GA-R2 & TAACCCCCGTCAGCAGCAATTGACGCGTTGAAGTTATATT \\
\hline & MBP-164-GA-F1 & AATATAACTTCAACGCGTCAGCTGACGGGGGTTATGCGTT \\
\hline & MBP-164-GA-R1 & TAAAGACGTTATAAGATGCATCAGCAGCAATCAGCGGCCA \\
\hline & MBP-164-GA-F2 & TGGCCGCTGATTGCTGCTGATGCATCTTATAACGTCTTTA \\
\hline & MBP-164-GA-R2 & AACGCATAACCCCCGTCAGCTGACGCGTTGAAGTTATATT \\
\hline & PyronicSF-VSTx2-GA-F1 & AGCTGGAGTACAATVSTVSTTATTCTCGGCGCGAAATGCT \\
\hline & PyronicSF-SNAx2-GA-R1 & TCGGCCTTGATATAGACGTTTNSTNSCAGGAGCTCAAAAT \\
\hline & PyronicSF-SNAx2-GA-F2 & ATTTTGAGCTCCTGSNASNAAACGTCTATATCAAGGCCGA \\
\hline & PyronicSF-VSTx2-GA-R2 & AGCATTTCGCGCCGAGAATAASBASBATTGTACTCCAGCT \\
\hline & PyronicSF-VE-SS-GA-F1 & AGCTGGAGTACAATAGTAGTTATTCTCGGCGCGAAATGCT \\
\hline & PyronicSF-VE-SS-GA-R1 & TTGATATAGACGTTTTCTACCAGGAGCTCAAAATTCTGTCT \\
\hline & PyronicSF-VE-SS-GA-F2 & ATTTTGAGCTCCTGGTAGAAAACGTCTATATCAAGGCCGA \\
\hline & PyronicSF-VE-SS-GA-R2 & TCGCGCCGAGAATAACTACTATTGTACTCCAGCTTGTGAC \\
\hline \multirow{20}{*}{ 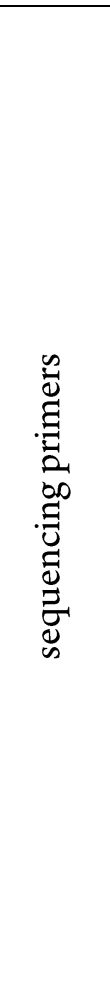 } & PyronicSF-LLseq-F & ACACTCTTTCCCTACACGACGCTCTTCCGATCTNNNNNACGTGAGACAGAATTTTG \\
\hline & PyronicSF-LLseq-R & TGACTGGAGTTCAGACGTGTGCTCTTCCGATCTNNNNNTAGACACGAGTGGCAGC \\
\hline & i5-IPE2p & AATGATACGGCGACCACCGAGATCTACACACACTCTTTCCCTACACGAC \\
\hline & i7-TXX-iPE2p & CAAGCAGAAGACGGCATACGAGAT[i7 index]GTGACTGGAGTTCAGACGTGTGC \\
\hline & i7-T71-IPE2p & CAAGCAGAAGACGGCATACGAGATaactaggcgcGTGACTGGAGTTCAGACGTGTGC \\
\hline & i7-T72-IPE2p & CAAGCAGAAGACGGCATACGAGATtcgctaagcaGTGACTGGAGTTCAGACGTGTGC \\
\hline & i7-T73-IPE2p & CAAGCAGAAGACGGCATACGAGATtatatactaaGTGACTGGAGTTCAGACGTGTGC \\
\hline & i7-T74-IPE2p & CAAGCAGAAGACGGCATACGAGATacttgctagaGTGACTGGAGTTCAGACGTGTGC \\
\hline & i7-T75-IPE2p & CAAGCAGAAGACGGCATACGAGATaaccattggaGTGACTGGAGTTCAGACGTGTGC \\
\hline & i7-T76-IPE2p & CAAGCAGAAGACGGCATACGAGATtcgcggttggGTGACTGGAGTTCAGACGTGTGC \\
\hline & i7-T77-IPE2p & CAAGCAGAAGACGGCATACGAGATcgtagttaccGTGACTGGAGTTCAGACGTGTGC \\
\hline & i7-T78-IPE2p & CAAGCAGAAGACGGCATACGAGATtccaatcatcGTGACTGGAGTTCAGACGTGTGC \\
\hline & i7-T79-IPE2p & CAAGCAGAAGACGGCATACGAGATaatcgataatGTGACTGGAGTTCAGACGTGTGC \\
\hline & i7-T80-IPE2p & CAAGCAGAAGACGGCATACGAGATccattatctaGTGACTGGAGTTCAGACGTGTGC \\
\hline & i7-T81-IPE2p & CAAGCAGAAGACGGCATACGAGATtcaacgtaagGTGACTGGAGTTCAGACGTGTGC \\
\hline & i7-T82-IPE2p & CAAGCAGAAGACGGCATACGAGATtctaatagtaGTGACTGGAGTTCAGACGTGTGC \\
\hline & i7-T83-IPE2p & CAAGCAGAAGACGGCATACGAGATaaccgctggtGTGACTGGAGTTCAGACGTGTGC \\
\hline & i7-T84-IPE2p & CAAGCAGAAGACGGCATACGAGATgatcgcttctGTGACTGGAGTTCAGACGTGTGC \\
\hline & i7-T85-IPE2p & CAAGCAGAAGACGGCATACGAGATctaactagatGTGACTGGAGTTCAGACGTGTGC \\
\hline & i7-T86-IPE2p & CAAGCAGAAGACGGCATACGAGATgctggaacttGTGACTGGAGTTCAGACGTGTGC \\
\hline
\end{tabular}




\begin{tabular}{|c|c|c|}
\hline \multirow{12}{*}{ 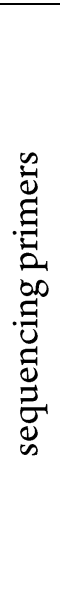 } & i5-NEX2p & AATGATACGGCGACCACCGAGATCTACACGTCTCGTGGGCTCGGAGATG \\
\hline & i7-TXX-NEX2p & CAAGCAGAAGACGGCATACGAGAT[i7 index]GTCTCGTGGGCTCGGAGATG \\
\hline & i7-T71-NEX2p & CAAGCAGAAGACGGCATACGAGATaactaggcgcGTCTCGTGGGCTCGGAGATG \\
\hline & i7-T72-NEX2p & CAAGCAGAAGACGGCATACGAGATtcgctaagcaGTCTCGTGGGCTCGGAGATG \\
\hline & i7-T73-NEX2p & CAAGCAGAAGACGGCATACGAGATtatatactaaGTCTCGTGGGCTCGGAGATG \\
\hline & i7-T74-NEX2p & CAAGCAGAAGACGGCATACGAGATacttgctagaGTCTCGTGGGCTCGGAGATG \\
\hline & i7-T75-NEX2p & CAAGCAGAAGACGGCATACGAGATaaccattggaGTCTCGTGGGCTCGGAGATG \\
\hline & i7-T76-NEX2p & CAAGCAGAAGACGGCATACGAGATtcgcggttggGTCTCGTGGGCTCGGAGATG \\
\hline & i7-T77-NEX2p & CAAGCAGAAGACGGCATACGAGATcgtagttaccGTCTCGTGGGCTCGGAGATG \\
\hline & i7-T78-NEX2p & CAAGCAGAAGACGGCATACGAGATtccaatcatcGTCTCGTGGGCTCGGAGATG \\
\hline & i7-T79-NEX2p & CAAGCAGAAGACGGCATACGAGATaatcgataatGTCTCGTGGGCTCGGAGATG \\
\hline & i7-T80-NEX2p & CAAGCAGAAGACGGCATACGAGATccattatctaGTCTCGTGGGCTCGGAGATG \\
\hline$\frac{\sqrt{u}}{0}$ & MBP 1-370 & $\begin{array}{l}\text { gcaggctccaccatggggcatatgtccAAAATCGAAGAAGGTAAACTGGTAATCTGGATTAACGGCGA } \\
\text { TAAAGGCTATAACGGACTCGCTGAAGTCGGTAAGAAATTCGAGAAAGATACCGGAAT } \\
\text { TAAAGTCACCGTTGAGCATCCGGATAAACTGGAAGAGAAATTCCCACAGGTTGCGG } \\
\text { CAACTGGCGATGGCCCTGACATTATCTTCTGGGCACACGACCGCTTTGGTGGCTAC } \\
\text { GCTCAATCTGGCCTGTTGGCTGAAATCACCCCGGACAAAGCGTTCCAGGACAAGCT } \\
\text { GTATCCGTTTACCTGGGATGCCGTACGTTACAACGGCAAGCTGATTGCTTACCCGA } \\
\text { TCGCTGTTGAAGCGTTATCGCTGATTTATAACAAAGATCTGCTGCCGAACCCGCCA } \\
\text { AAAACCTGGGAAGAGATCCCGGCGCTGGATAAAGAACTGAAAGCGAAAGGTAAGAG } \\
\text { CGCGCTGATGTTCAACCTGCAAGAACCGTACTTCACCTGGCCGCTGATTGCTGCTG } \\
\text { ACGGGGGTTATGCGTTCAAGTATGAAAACGGCAAGTACGACATTAAAGACGTGGGC } \\
\text { GTGGATAACGCTGGCGCGAAAGCGGGTCTGACCTTCCTGGTTGACCTGATTAAAAA } \\
\text { CAAACACATGAATGCAGACACCGATTACTCCATCGCAGAAGCTGCCTTTAATAAAGG } \\
\text { CGAAACAGCGATGACCATCAACGGCCCGTGGGCATGGTCCAACATCGACACCAGCA } \\
\text { AAGTGAATTATGGTGTAACGGTACTGCCGACCTTCAAGGGTCAACCATCCAAACCG } \\
\text { TTCGTTGGCGTGCTGAGCGCAGGTATTAACGCCGCCAGTCCGAACAAAGAGCTGGC } \\
\text { GAAAGAGTTCCTCGAAAACTATCTGCTGACTGATGAAGGTCTGGAAGCGGTTAATAA } \\
\text { AGACAAACCGCTGGGTGCCGTAGCGCTGAAGTCTTACGAGGAAGAGTTGGCGAAAG } \\
\text { ATCCACGTATTGCCGCCACCATGGAAAACGCCCAGAAAGGTGAAATCATGCCGAACA } \\
\text { TCCCGCAGATGTCCGCTTTCTGGTATGCCGTGCGTACTGCGGTGATCAACGCCGCC } \\
\text { AGCGGTCGTCAGACTGTCGATGAAGCCCTGAAAGACGCGCAGACTCGTATCACCaag } \\
\text { agcggtcaccatcaccatcaccattaagcggccgcactcgagatatcta }\end{array}$ \\
\hline
\end{tabular}

